unnecessary hospital admissions and burdensome interventions. Research is needed to inform our understanding of how ACP works in practice. Accordingly, a two phased study is currently engaged in the Care Home setting, which seeks to:

Develop a consensual process for ACP

Assess the impact that ACP discussions have on all participants; residents, relatives/friends and healthcare professionals.

Findings from phase 1 of the study indicated that existing definitions of ACP are complex, and understanding was varied. A recommendation for this study was that the term Anticipatory Care Planning (AnCP) would be used to describe the planning process. Phase 2 of this project is now underway.

Aim(s) To examine the lived experience of relative/friends, who have been engaged in conversations about AnCP, for residents living in care homes.

Method(s) Interpretive phenomenology underpin the research methods employed; In-depth semi-structured interviews with residents (where possible) and relatives/friends of care home residents, are being undertaken using a longitudinal case study design:

Initial interview after first AnCP discussion

Follow up interview(s) at 3 monthly intervals throughout the study period

Post bereavement interview with relative/friends and healthcare professionals.

Results Qualitative data derived from the interviews will be discussed under the following emerging themes:

Timing and content of discussions

Emotional impact of engaging in discussions

Confidence in care delivery in care home setting

Conclusion(s) Conclusions will inform the ongoing debate and understanding around the broader concept of Advance Care Planning, and also the impact that these types of discussions may have on residents and their relatives/friends.

\section{P 006 ANTICIPATORY CARE PLANNING: UNDERSTANDING THE IMPACT THAT TALKING ABOUT PLANNING FOR FUTURE CARE HAS ON RELATIVES/FRIENDS OF CARE HOME RESIDENTS}

Tamsin McGlinchey, ${ }_{1}^{1}$ Philip Saltmarsh, ${ }^{1}$ Rebecca Bancroft, ${ }^{2}$ Stephen Mason, ${ }^{1}$ Gerard Corcoran, ${ }^{1}$ John Ellershaw'. 'Marie Curie Palliative Care Institute Liverpool, Liverpool, UK; ${ }^{2}$ Royal Liverpool \& Broadgreen University Hospital NHS Trust, Liverpool, UK

\subsection{6/bmjspcare-2014-000654.46}

Background Advance Care Planning (ACP) has become increasingly important in terms of its perceived role in improving care planning for people nearing End of Life (EOL). ACP can enable people to die in their place of choice, coordinate care, reduce 